\title{
Potential energy landscape formalism for quantum liquids
}

\author{
Nicolas Giovambattista $\oplus^{1,2, *}$ and Gustavo E. Lopez $\oplus^{2,3, \uparrow}$ \\ ${ }^{1}$ Department of Physics, Brooklyn College of the City University of New York, Brooklyn, New York 11210, USA \\ ${ }^{2}$ The Graduate Center of the City University of New York, New York, New York 10016, USA \\ ${ }^{3}$ Department of Chemistry, Lehman College of the City University of New York, Bronx, New York 10468, USA
}

(Received 12 September 2020; accepted 8 December 2020; published 31 December 2020)

\begin{abstract}
The potential energy landscape (PEL) formalism is a theoretical approach within statistical mechanics used extensively in the past to study classical liquids and glasses. Here, we extend the PEL formalism to the case of quantum liquids. As an example, we apply the PEL approach to study a family of quantum monatomic liquids using path-integral Monte Carlo simulations. We focus on the energy $\left(E_{\mathrm{IS}}\right)$ and pressure $\left(P_{\mathrm{IS}}\right)$ of the local minima of the PEL [inherent structures (IS)] explored by the liquids. It is found that, similar to the classical case, the quantum liquids exhibit a PEL-independent regime at high temperatures and a PEL-influenced regime at low temperatures, where the topography of the PEL plays a major role. Interestingly, the PEL of all the quantum liquids studied is Gaussian, providing a simple expression for the configurational entropy of the liquids. Remarkably, the ring-polymers representing the atoms of the quantum liquids are collapsed at the IS. Accordingly, an IS of the quantum liquid, in its own PEL, is also an IS of the classical liquid in the classical liquid PEL (CL-PEL). A pictorial interpretation of the behavior of quantum liquids using the CL-PEL (as opposite to the quantum liquid PEL) is provided. In this view, the quantum liquid is represented by a pancakelike patch that expands over multiple IS of the CL-PEL, changing shape with time while describing a fuzzy trajectory (on the CL-PEL). The formalism described in this work is general and can, in principle, be extended to quantum systems other than liquids.
\end{abstract}

DOI: 10.1103/PhysRevResearch.2.043441

\section{INTRODUCTION}

As a liquid is supercooled toward its glass state, the liquid's dynamics slows down abruptly, with relaxation times increasing by $\approx 10-15$ orders of magnitude in a small temperature range $[1,2]$. Understanding the behavior of liquids at low temperature and the nature of the associated glass state has been a fundamental problem in condensed-matter physics and material science for decades [3-13], with numerous important applications (see, e.g., Refs. [1,4]). In most of these studies, the liquids of interest are classical. This is because in most cases, the glass transition temperature is relatively high and/or because the liquid is composed of heavy molecules/atoms.

Although the slowdown of most known supercooled liquids approaching the glass state can be explained using classical theories [e.g., mode coupling theory (MCT) [10]], quantum effects can play a relevant role in the case of light elements, including $\mathrm{H}_{2}$ and $\mathrm{He}[14,15]$. Nuclear quantum effects are known to occur even for the case of water [16-18]; for example, the glass transition temperature of $\mathrm{H}_{2} \mathrm{O}$ and $\mathrm{D}_{2} \mathrm{O}$ differs by $\approx 10 \mathrm{~K}$ [17]. There are also systems that are intrinsically quantum in nature that also exhibit slow or

\footnotetext{
*Corresponding author: ngiovambattista@brooklyn.cuny.edu

${ }^{\dagger}$ Corresponding author: gustavo.lopez1@lehman.cuny.edu
}

Published by the American Physical Society under the terms of the Creative Commons Attribution 4.0 International license. Further distribution of this work must maintain attribution to the author(s) and the published article's title, journal citation, and DOI. glassy dynamics and even a glass transition. Examples include electronic [19] and magnetic (spin) systems [20]. It is not clear how the laws of quantum mechanics may affect such systems as they slowdown toward the glass state. In some cases, quantum effects can lead to novel phenomena, such as the existence of a "superglass" state $[15,21,22]$, in which a system exhibits superfluidity coexisting with dynamically arrested domains. In other cases, it may be that quantum fluctuations destroy the glass state [23].

At present, a unifying theory that explains the behavior of quantum glass forming liquids is not available. Intuitively, one would expect that the inclusion of quantum fluctuations should suppress the glass state via, e.g., tunneling and zero-point energy fluctuations, which may allow particles to overcome energy barriers. Indeed, experiments in spin glasses show that adding quantum fluctuations provides additional paths to relaxation, lowering the glass transition temperature [20]. However, other studies on spin glasses and liquids indicate that the addition of quantum fluctuations can have a counterintuitive effect on the dynamics of the glass forming system where the quantum system exhibits a slower dynamics than its classical counterpart [24,25]. A microscopic dynamical theory, based on the classical MCT, has been developed for the description of quantum liquids as they approach the glass state [26]. In particular, the resulting quantum MCT predicts novel effects, such as the counterintuitive slowing down of the liquid dynamical relaxation times with the inclusion of quantum fluctuations.

In this work, we extend the potential energy landscape (PEL) formalism, originally proposed to describe the behav- 

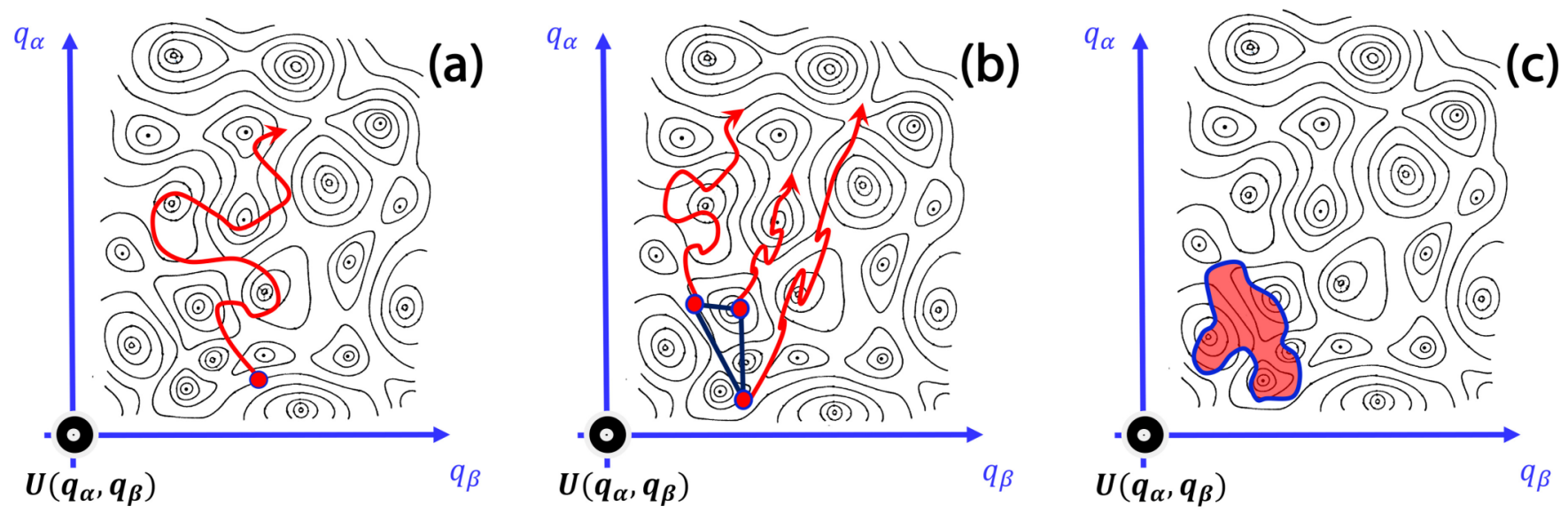

FIG. 1. (a) Schematic representation of the PEL of a classical monatomic liquid composed of $N$ atoms. $q_{\alpha}$ and $q_{\beta}$ represent the $3 N$ generalized coordinates of the system, $\alpha, \beta=1,2,3,4, \ldots, 3 N$, with $\alpha \neq \beta ; U\left(q_{\alpha}, q_{\beta}\right)$ is the corresponding potential energy that defines the PEL. Contour lines represent constant potential energy levels. The PEL is partitioned into basins; the associated local minima, i.e., the IS, are indicated by dots. The system is represented by the red dot, and the corresponding trajectory (over time) is indicated by a red line. Adapted from Ref. [42]. (b) Schematic representation of a quantum liquid composed of $N$ atoms, represented by a (classical) ring-polymer system with $n_{b}=3$ beads. In this case, the PEL of the classical liquid [CL-PEL, from (a)] is used. Each replica is represented by a different red dot, and the corresponding trajectory is indicated by a red path on the CL-PEL. (c) Schematic representation of a quantum liquid composed of $N$ atoms in the limit $n_{b} \rightarrow \infty$. In this case, the system is represented by a pancakelike patch that spreads over many configurations of the CL-PEL. The representative patch of the system evolves with time, changing shape and describing a fuzzy trajectory on the CL-PEL.

ior of classical low-temperature liquids and glasses, to the case of quantum liquids. The PEL formalism is based solely on statistical mechanics and it has been successfully applied to the description of realistic (classical) liquids, including water and silica [27,28], and glasses, such as amorphous ice [29-32]. The PEL has also been used to study clusters of atoms, biomolecules [33], and low-density condensedmatter systems [34]. Applications of the PEL formalism are also found in the study of out-of-equilibrium liquids [35] and in the calculation of the equation of state of equilibrium systems [36-38]. We note that the PEL approach is well-established with its original ideas traced back to the work of Goldstein in the 1970s [39,40] (and others; see, e.g., Ref. [38]). It was Weber and Stillinger in the 1980s who set the PEL formalism on firm theoretical grounds and showed the power of this approach when combined with computer simulations [41-43].

This article is organized as follows. In Sec. II, we provide a brief introduction of the PEL formalism to the case of classical systems, and we discuss the method followed to extend it to quantum liquids. Section III includes the computational details and model liquids employed in this work. The results are presented in Sec. IV. A summary and discussion are included in Sec. V.

\section{DEFINING A PEL FOR A QUANTUM SYSTEM}

For a classical system composed of $N$ particles in a volume $V=L_{x} \times L_{y} \times L_{z}$, the PEL is the hypersurface in $(3 N+1)$-dimensional space defined by the potential energy of the system as function of all $3 N$-coordinates, $U\left(\vec{r}_{1}, \vec{r}_{2}, \ldots, \vec{r}_{N}\right)$ [37,39-43]; see Fig. 1(a). For simplicity, we are considering here the particle positions $\vec{r}_{i}=\left(x_{i}, y_{i}, z_{i}\right)$ $\left(i=1,2, \ldots, N ; 0 \leqslant x_{i} \leqslant L_{x}, 0 \leqslant y_{i} \leqslant L_{y}, 0 \leqslant z_{i} \leqslant L_{z}\right)$ to characterize the state of the system, but any other set of
$3 N$ generalized coordinates can be used. The PEL of a classical liquid is independent of temperature, and for a system at constant $N$ and $V$ the PEL defines the system. At any given time $t$, the system is represented by a single point on the PEL given by the coordinates of the particles at time $t$, $\left\{\vec{r}_{1}(t), \vec{r}_{2}(t), \ldots, \vec{r}_{N}(t)\right\}$. Hence, as time goes on, the representative point of the system evolves, describing a trajectory on the PEL [Fig. 1(a)]. At high temperatures, the representative point of the system can move freely on the PEL overcoming potential energy barriers and moving from one basin of the PEL to another. As temperature decreases, it becomes more difficult for the representative point of the system to overcome certain potential energy barriers, hence the regions of the PEL accessible to the system become limited. At very low temperature, in the glass state, the system loses ergodicity and it is able to move within single basins of the PEL.

The local minima of the PEL are called "inherent structures" (IS), and they play a fundamental role in the PEL formalism. For example, IS can be used to label the basins of the PEL; a basin is the set of all points of the PEL that lead to the same IS upon potential energy minimization [37]; see Fig. 1(a). There are other properties of the IS explored by the system at the given working condition that play a relevant role. These include the average energy and pressure of the system at the IS, $E_{\mathrm{IS}}(T)$ and $P_{\mathrm{IS}}(T)$, the distribution of IS energies within the PEL, and the average curvature of the PEL at the IS. The curvature of the PEL at a given IS can be quantified by the so-called shape function $\mathcal{S}_{\text {IS }}$. $\mathcal{S}_{\text {IS }}$ is a function of the $6 N$ eigenvalues of the Hessian matrix evaluated at the IS of interest [38]. It can be shown that, under simple assumptions, the Helmholtz free energy of the system $F(N, V, T)$ can be written in terms of the distribution of IS energies within the PEL, $E_{\mathrm{IS}}, P_{\mathrm{IS}}$, and $\mathcal{S}_{\mathrm{IS}}$, where again $E_{\mathrm{IS}}, P_{\mathrm{IS}}$, and $\mathcal{S}_{\mathrm{IS}}$ are average properties that depend on the IS explored by the system at the working conditions, e.g., $E_{\mathrm{IS}}=E_{\mathrm{IS}}(N, V, T)$ [38]. 
To make a connection between the PEL formalism, defined for classical systems, and the thermodynamic/statistical mechanics description of a quantum system, we consider the path integral formulation of statistical mechanics. Within the path integral approach, it can be shown that the canonical partition function of a quantum system (e.g., liquid, solid) at temperature $T$ is identical to the canonical partition function of a classical system composed of ring-polymers [44,45]. Specifically, each atom of the quantum system is replaced by a ring-polymer composed of $n_{b}$ beads where $n_{b} \rightarrow \infty$. The beads of a given ring-polymer are connected by springs with $T$-dependent spring constant $k_{\text {sp }} \propto(T / h)^{2}$, where $h$ is Planck's constant (see Sec. III). At high $T$ the spring constants are very large, and hence the ring-polymers collapse to a single point, i.e., the quantum/ring-polymer system reduces to a classical system of point particles. The beads of a given ring-polymer are numbered $n=1,2,3, \ldots, n_{b}$, and in the path integral formalism of statistical mechanics, the bead $n$ of polymer A interacts only with bead $n$ of polymer B (bead $n$ of polymer A does not interact with bead $m \neq n$ of polymer B). Moreover, the interaction between beads $n$ of polymers $\mathrm{A}$ and $\mathrm{B}$ is given by the same formal expression of the potential energy function of the quantum atoms (defined by its Hamiltonian operator) but rescaled by a factor $1 / n_{b}$ (see Sec. III).

It follows that one can apply the PEL formalism to the classical ring-polymer system and then use this information to describe the thermodynamic/statistical mechanical properties of the corresponding quantum liquid. For example, one could in principle be able to use the PEL formalism to obtain the $F(N, V, T)$ of the ring-polymer system, and by doing so, obtain the Helmholtz free energy of the quantum system. After all, the free energies $F(N, V, T)$ of the quantum and ring-polymer systems converge toward each other with increasing $n_{b}$ and become identical in the limit $n_{b} \rightarrow \infty$ [44]. We note that the PEL formalism was originally proposed to study low-temperature systems, including supercooled liquids and glasses, and hence it is well-suited for the study of quantum systems; quantum effects are usually relevant at low temperatures. We also stress that the "isomorphism" between the given quantum system at temperature $T$ and its associated classical ring-polymer system with spring constant $k_{\mathrm{sp}} \propto$ $(T / h)^{2}$ is limited to equilibrium conditions. Accordingly, one may apply the PEL approach to study quantum systems in equilibrium, metastable equilibrium, and quasiequilibrium. This excludes the glass state. Yet, the ideas of the PEL formalism may open a door to understand how a quantum liquid enters the glass state, and perhaps it can also provide insight into the behavior of quantum glasses.

There is a subtle difference between classical liquids and quantum liquids that one must take into consideration when applying the PEL approach. In classical liquids, the PEL is necessarily $T$-independent since, after all, the potential energy of a classical system cannot depend on $T$. In the case of a quantum liquid, this is not the case. At temperature $T_{1}$, the quantum liquid is isomorphic to the ring-polymer system with spring constants $k_{\mathrm{sp}}^{1} \propto\left(T_{1} / h\right)^{2}$, while at temperature $T_{2}$ the quantum liquid is isomorphic to a different ring-polymer system with spring constants $k_{\mathrm{sp}}^{2} \propto\left(T_{2} / h\right)^{2}$. Accordingly, the PEL associated with the quantum liquid is indeed
$T$-dependent. This has profound implications in the description of the quantum liquid using the PEL approach since it implies that, e.g., potential energy barriers may increase/decrease, and even vanish, depending on the $T$ considered.

To explore the ideas presented above, in this work we consider a monatomic quantum liquid. Our main goal is to characterize the PEL of this ring-polymer/quantum system at constant volume and explore the behavior of $E_{\mathrm{IS}}$ and $P_{\mathrm{IS}}$ as the system gets colder and approaches the glass state. In particular, by tuning the value of Planck's constant, we will describe how the PEL of the classical liquid ( $h=0$ or, equivalently, $k_{\mathrm{sp}} \rightarrow \infty$ ) evolves as the liquid becomes more and more quantum $(h>0)$.

By definition, the PEL of the ring-polymer system is a hypersurface in $\left[3\left(N \times n_{b}\right)+1\right]$-dimensional space defined by the potential energy of the ring-polymer system as a function of the $3\left(N \times n_{b}\right)$ coordinates of the beads, $\left\{\vec{r}_{i, k}\right\}$; here, $i=1,2, \ldots, N$ is the ring-polymer number and $k=$ $1,2, \ldots, n_{b}$ is the corresponding bead number. Again, the ring-polymer system is represented by a single point in its PEL. Qualitatively, the PEL of the ring-polymer system is also given by Fig. 1(a) but with $\alpha, \beta=1,2, \ldots, 3 N n_{b}(\alpha \neq$ $\beta$ ). Interestingly, it is also possible to consider the PEL of the classical liquid to describe the behavior of the quantum liquid/ring-polymer system. In the classical liquid PEL (CLPEL), the quantum liquid can be represented by $n_{b}$ points; see Fig. 1(b). These $n_{b}$ points in the CL-PEL are given by the socalled "replicas." Specifically, replica $k\left(k=1,2,3, \ldots, n_{b}\right)$ is defined by the set of $3 N$ coordinates $\left\{\vec{r}_{i, k}\right\}$, where $i=$ $1,2, \ldots, N$ and $k$ is fixed (alternatively, replica $k$ is composed of beads $k$ of all $N$ ring-polymers in the system). It follows that, in the CL-PEL, the system is represented by a "cloud" composed of $n_{b}$ points that move with time, describing collectively a fuzzy path in the CL-PEL. In the limit $n_{b} \rightarrow \infty$, such a "cloud" would represent a continuous pancakelike patch that moves and changes shape with time; see Fig. 1(c). In most of this work, we will describe the quantum system using the PEL of the ring-polymer system (briefly, the "PEL" [Fig. 1(a)]). In Sec. IV E, we will also interpret the results of our path-integral Monte Carlo simulations in terms of the CL-PEL [Figs. 1(b) and 1(c)] and show that this alternative PEL-based approach can be very insightful to describe the behavior of the quantum liquid at low temperatures.

\section{COMPUTER SIMULATIONS}

We perform path-integral Monte Carlo (PIMC) simulations of a monatomic system at a constant volume and a wide range of temperatures. The Fermi-Jagla $(\mathrm{FJ})$ pair potential $U_{\mathrm{FJ}}(r)$ is used to represent the interactions between atoms of the quantum liquid. As shown in Fig. S1 of the Supplemental Material (SM) [46], this is a core-softened pair-potential with a hard-core radius $r=a$, an attractive part centered at $r=b \approx$ $2 a$, and a soft-core part that extends approximately over the range $a<r<b$; see also Ref. [47]. In PIMC simulations, the beads of a given ring-polymer are connected by springs with spring constant $k_{\mathrm{sp}}=4 \pi^{2} m n_{b} /(h \beta)^{2}$, where $\beta=1 / k_{B} T$, and $k_{B}$ is Boltzmann's constant [44]. In addition, the pair potential between an interacting pair of beads is given by the same 
pair-interaction potential included in the Hamiltonian of the the original quantum system, $U_{\mathrm{FJ}}(r)$, but rescaled by a factor $1 / n_{b}$ [i.e., in the case of our quantum Fermi-Jagla liquids, the pair potential between interacting beads is given by $U_{\mathrm{FJ}}(r) / n_{b}$, where $r$ is the corresponding bead-bead distance].

The FJ model can reproduce qualitatively many of water's anomalous properties in the liquid state as well as a water glass-glass first-order-like phase transition at low temperatures [47-53]. In addition, this model liquid exhibits a liquid-liquid phase transition ending at a (liquid-liquid) critical point, as it has been hypothesized for the case of supercooled water [54-57] and reported in computational studies of a few classical water models [54,58]. In a previous work [51], we studied the thermodynamic behavior of the Fermi-Jagla liquid by performing PIMC simulations $\left(n_{b}=10\right)$ with different values of $h$. Changing $h$ affects the value of $k_{\mathrm{sp}}$, resulting in a family of quantum Fermi-Jagla (QFJ) liquids where the level of "quantumness" increases with increasing $h$ (the atoms' mass is kept constant, $m=1)$. It was shown that, as the liquids evolve from classical $(h=0)$ to increasingly quantum $(h>0)$, the location of the liquid-liquid critical point (LLCP) in the P-T plane shifts toward lower temperatures and larger pressures until crystallization interferes at large $h$ [51]. In addition, the anomalous properties of the liquid and the liquidliquid phase transition (LLPT) can be drastically affected as the atoms become more delocalized, i.e., as $h$ increases [52]. Remarkably, the LLCP volume remains $h$-independent, equal to the LLCP volume of the classical liquid, $v_{c}=V_{c} / N \approx$ 2.9 [51,52]. To avoid any LLPT upon cooling, all our simulations in this work are performed at $v=2.2<v_{c}$. At this volume, the system remains in the so-called high-density liquid state at all temperatures. In particular, in the case of the classical FJ liquid, the crystallization temperature reaches a minimum at $v \approx 2.2-2.3$. Hence, working at $v=2.2$ allows us to explore the liquid state at relatively low temperatures. We note that MD simulations of the classical FJ liquid show that, at $v=2.2$, one can observe a mild two-step ( $\alpha$ and $\beta$ ) relaxation [50]. It is this ability to exhibit two-step relaxation that motivates us to use the (monatomic, one-component) FJ model liquid. This is unusual since in most monatomic liquids, crystallization occurs so rapidly that a two-step relaxation, which is a feature of good glass formers, cannot be observed. For example, in order to avoid crystallization in Lennard-Jones (LJ) liquids, one needs to consider a binary mixture of particles with different LJ parameters [5].

We use the same PIMC simulation techniques as in Refs. [51,52] and refer the reader to these works for details. Most of our PIMC simulations are performed with $n_{b}=10$; simulations with $n_{b}=5,15,20$ are also performed to test the sensitivity of our results to $n_{b}$. Simulations are performed for systems with $N=512\left(n_{b}=15,20\right)$ and $N=1000$ atoms $\left(n_{b}=5,10\right)$ in a cubic box. We use reduced units defined by setting the particle mass $m=1$ and $k_{B}=1$. Energies are given in units of $\epsilon_{0}$ [see the definition of $U_{\mathrm{FJ}}(r)$ in SM [46]]; distances are given in units of $a$. It follows that the units of $h$ are $a\left(\epsilon_{0} m\right)^{1 / 2}$. The values of Planck's constant studied in this work are $h_{0}=0$ (classical system with $n_{b}=1$ ), $h_{1}=0.2474$, $h_{2}=0.5150, h_{3}=0.7948$, and $h_{4}=1.7882$. To understand the level of quantumness of the resulting QFJ liquids, we compare the corresponding de Broglie's thermal wavelength, $\lambda=h / \sqrt{2 \pi k_{B} m T}$, for the case of the light molecule $\mathrm{H}_{2}$ [51]. At $T=10$ and $300 \mathrm{~K}$, one gets $(m=2 \times 1.008 \mathrm{~g} / \mathrm{mol}) \lambda=$ 3.9 and $0.71 \AA$, respectively. Assuming that $a$ is close to the nearest-neighbors distance in $\mathrm{H}_{2}$ [59], $a \approx 3.2 \AA$, we get $\lambda=1.22$ and 0.22 in reduced units. In our PIMC simulations, $\lambda_{\text {PIMC }}=h / \sqrt{2 \pi T}$, where $h$ and $T$ are in reduced units and $\lambda_{\text {PIMC }}$ is in units of $a$. Since in our PIMC simulations $T \approx$ 0.10 , it follows that for $h_{1} \leqslant h \leqslant h_{4}, 0.31 \leqslant \lambda_{\text {PIMC }} \leqslant 2.25$. These values are comparable to the values of $\lambda$ estimated for $\mathrm{H}_{2}$ at $T=10-300 \mathrm{~K}$. Similarly, one can estimate the value of $h$ in reduced units, i.e., $h=6.626 \times 10^{-34} \mathrm{~J} \mathrm{~s} /\left[a\left(\epsilon_{0} m\right)^{1 / 2}\right]$, for the case of $\mathrm{H}_{2}$. An approximate pair interaction LennardJones potential has been proposed for the $\mathrm{H}_{2}$ molecular gas based on thermodynamic calculations (see Ref. [59] and references therein). This LJ potential has a potential energy minimum depth of approximately $40.3 \times 10^{-23} \mathrm{~J}$. Using this value for $\epsilon_{0}$ (and approximating $a=3.2 \AA$ ), we obtain that $h \approx 1.78$ in reduced units. This value is within the range of $h$-values considered in this work and approximately the same value of $h_{4}$. Similarly, if one considers a system of He atoms, one obtains $h \approx 4.57\left(\epsilon_{0}=14.9 \times 10^{-23} \mathrm{~J}\right.$ and $a=2.9 \AA$ [59]). For comparison, in the SM [46], we also include results for $h_{4 b}=3.5764$, corresponding to $\lambda_{\mathrm{PIMD}}=$ $4.51(T=0.10)$.

PIMC simulations are performed for $10^{6}$ steps during equilibration followed by another $10^{6}$ steps for data analysis. In one MC step, we first move all the $n_{b} \times N$ beads, and then the centroids of all $N$ ring-polymers are displaced. As shown in Sec. SI of the SM [46], this number of steps allows the system to reach equilibrium at sufficiently low temperatures. We consider that the system reaches equilibrium at a given temperature if its self-intermediate scattering function $F\left(k_{0}, n\right)$, as function of the number of PIMC simulation steps $n$, becomes zero for $n<10^{6}$; see Sec. I of the SM [46].

To calculate the PEL properties of the ring-polymer system, we obtain the IS of selected configurations by minimization of the potential energy using the conjugate gradient algorithm [50,60]. For given values of $(h, T)$, we select 100 IS obtained from configurations saved every $1000 \mathrm{MC}$ steps during the production runs. The average energy and pressure of the system over the obtained 100 IS define $E_{\mathrm{IS}}(T)$ and $P_{\mathrm{IS}}(T)$ (for the given value of $h$ ).

\section{RESULTS}

We first focus on two of the most relevant properties of the PEL, namely $E_{\mathrm{IS}}$ and $P_{\mathrm{IS}}$; see Sec. IV A. Our results are consistent with the PEL of the QFJ liquids being Gaussian. This allows us to obtain a general expression for the configurational entropy of the quantum liquids; see Sec. IV B. In Sec. IV C, we show that the ring-polymers are collapsed at the IS. The effects of varying $n_{b}$ on the PEL properties of the QFJ liquids are discussed in Sec. IV D. A description of the quantum liquids using the CL-PEL, as opposite to the ring-polymer system PEL, is included in Sec. IV E.

\section{A. Inherent structure energy and pressure}

Figure 2(a) shows $E_{\mathrm{IS}}(T)$ for the QFJ liquids with $h=$ $h_{0}, h_{1}, h_{2}, h_{3}$, and $h_{4}\left(n_{b}=10\right)$. Our values of $E_{\mathrm{IS}}(T)$ for $h=h_{0}$ (orange line) are fully consistent with the correspond- 


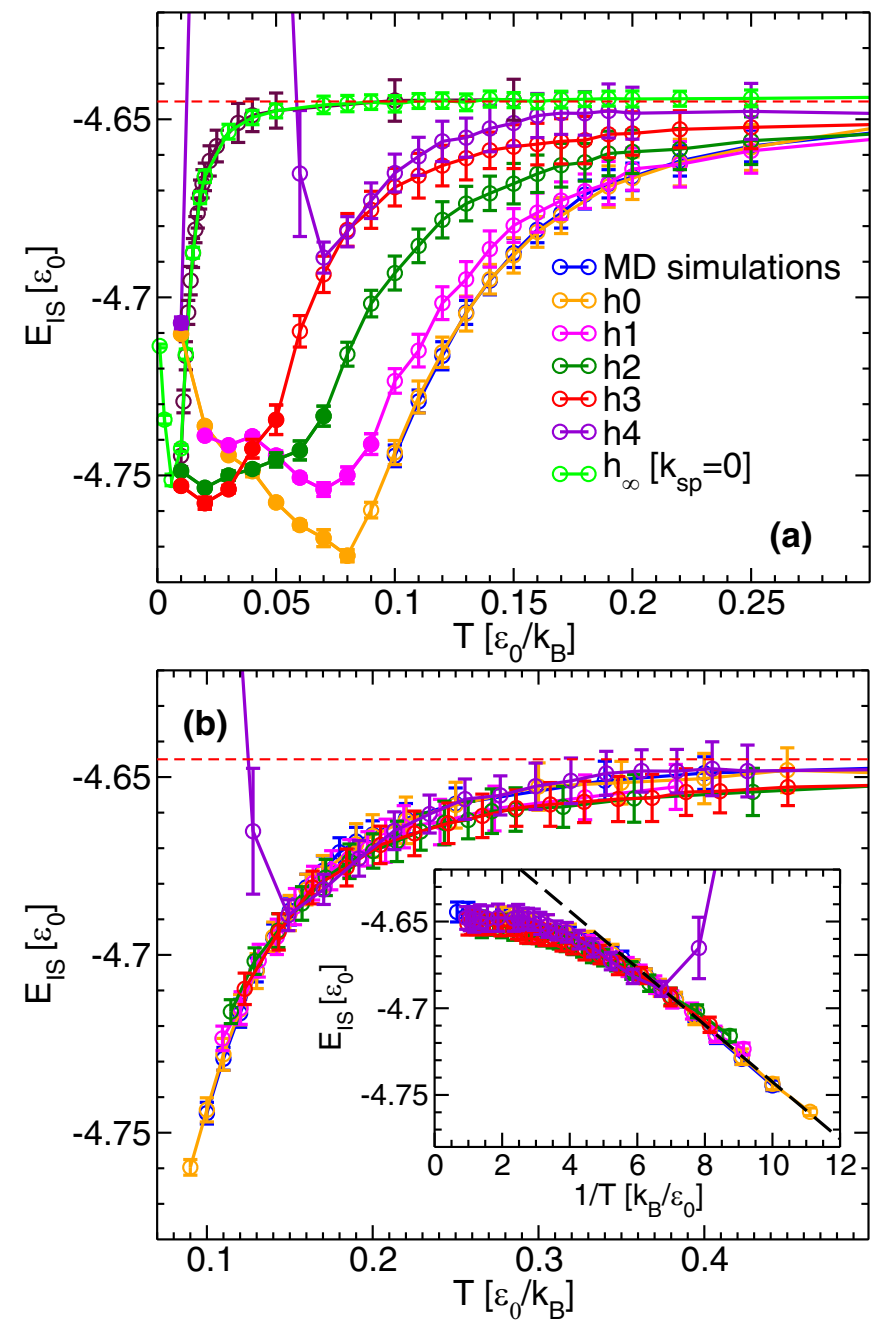

FIG. 2. (a) Inherent structure energy as a function of temperature for the FJ liquids with Planck's constant $h_{0}=0$ (classical system), $h_{1}=0.2474, h_{2}=0.5150, h_{3}=0.7948, h_{4}=1.7882$, and $h_{\infty}=\infty$ (spring constants $k_{\mathrm{sp}}=0.0$ ). Empty and solid symbols correspond to equilibrium and out-of-equilibrium states, respectively. Also included are results obtained from MD simulations for the classical FJ liquid from Ref. [50] (blue line). The maroon line is obtained from the classical $E_{\mathrm{IS}}(T)\left(h_{0}=0\right.$, orange line) by rescaling $T \rightarrow T / n_{b}$. In all cases, the number of beads per ring-polymer is $n_{b}=10$. A sudden increase in $E_{\mathrm{IS}}(T)$ occurs for the case $h=h_{4}$ at $T<0.07$; at these temperatures, results for $h=h_{4}$ are sensitive to $n_{b}$, and hence they are not reliable (see text and Fig. 6). (b) Within error bars, the $E_{\mathrm{IS}}(T)$ curves for the different QFJ liquids collapse onto the classical $E_{\mathrm{IS}}(T)$ curve if the temperature is rescaled by $T \rightarrow \gamma T$, where $\gamma=\gamma(h)$. Inset: $E_{\mathrm{IS}}(T)$, from the main panel, plotted as a function of $1 / T$. After $T$-rescaling, $E_{\mathrm{IS}}(T)=a-b / T(a=-4.5782, b=0.01643)$ at low temperatures (black dashed lines); see also Fig. 4.

ing values obtained in Ref. [50] for the classical FJ liquid using molecular dynamics (MD) simulations (blue line). Also included in Fig. 2(a) is the $E_{\mathrm{IS}}(T)$ for the QFJ liquid with $h=$ $h_{\infty}=\infty$. In the case of $h=h_{\infty}$, the spring constants of the ring-polymers vanish and the canonical partition function of the system can be evaluated analytically. As shown in Sec. II of the SM [46], the QFJ liquid with $h=h_{\infty}$ at temperature $T$ is thermodynamically identical to the classical FJ liquid
TABLE I. Scaling factor $\gamma$ used in Figs. 2(b) and 3(b) to shift the IS energy and pressure of the QFJ liquids along the temperature axis $(T \rightarrow \gamma T)$.

\begin{tabular}{lc}
\hline \hline$h\left[a\left(m \epsilon_{0}\right)^{1 / 2}\right]$ & $\gamma$ \\
\hline$h 1$ & 1.09368 \\
$h 2$ & 1.43077 \\
$h 3$ & 2.04710 \\
$h 4$ & 2.13022 \\
\hline \hline
\end{tabular}

( $\left.h=h_{0}\right)$ at temperature $T^{\prime}=n_{b} T$. Similarly, the PEL properties $E_{\mathrm{IS}}(T)$ and $P_{\mathrm{IS}}(T)$ of the quantum liquid with $h=h_{\infty}$ at temperature $T$ are identical to the PEL of classical liquid at temperature $T^{\prime}$ (see Sec. II of SM [46]). Accordingly, Fig. 2(a) shows that the $E_{\mathrm{IS}}(T)$ of the QFJ liquid with $h=h_{\infty}$ (green line), obtained from our PIMC simulations, is identical to the $E_{\mathrm{IS}}(T)$ of the classical FJ liquid (orange line) after rescaling the temperature $T \rightarrow T / n_{b}$ (maroon line). We note that while the qualitative behavior of $E_{\mathrm{IS}}(T)$ is similar for $h \leqslant h_{3}$, the case of $h=h_{4}$ is anomalous. Specifically, in the case $h=h_{4}$, $E_{\mathrm{IS}}(T)$ increases dramatically at $T<0.07$. As we discuss in Sec. IV D, the results obtained at $T<0.07$ for $h=h_{4}$ are sensitive to the number of beads per ring-polymer employed; for $h \geqslant h_{4}$ and $T<0.07$, one must use a value $n_{b}>10$.

In the case of classical liquids, $E_{\mathrm{IS}}(T)$ is constant at high temperatures in the so-called PEL-independent regime [61] At these temperatures, the properties of the system are not sensitive to the topography of the PEL; the system has relatively large kinetic energy, and hence it can freely explore the PEL. Instead, at low temperature, the thermodynamic properties of the system are strongly correlated with the topography of the PEL. This is the so-called PELinfluenced regime [61] where $E_{\mathrm{IS}}(T)$ varies nonlinearly with $T$. The crossover temperature between the PEL-independent and PEL-influenced regimes occurs at the onset temperature $T_{0}$ [50,61]. The main point of Fig. 2(a) is that all QFJ liquids exhibit PEL-independent and PEL-influenced regimes, same as classical liquids. In particular, the onset temperature decreases as the quantumness of the liquids (as quantified by $h$ ) increases and the atoms of the QFJ liquid become more delocalized.

We note that at approximately $T>0.25$, all QFJ liquids are in the PEL-independent regime and exhibit similar values of $E_{\mathrm{IS}}(T) \approx-4.65$. This is not surprising since at high temperatures, nuclear quantum effects become less relevant and all QFJ liquids must approach the classical limit $\left(h=h_{0}\right.$, orange line). What is remarkable is that the $E_{\mathrm{IS}}(T)$ for all the QFJ liquids collapse onto a unique curve if one rescales the temperature. This is shown in Fig. 2(b), where the $E_{\mathrm{IS}}(T)$ of the QFJ liquids is plotted as a function of a rescaled temperature $(T \rightarrow \gamma T$, where $\gamma$ depends only on $h$; see Table I). This strongly suggests that the same physical description of classical liquids, based on the PEL approach, as they approach their glass-to-liquid transition, also applies to the case of the quantum liquids. The effects of making the liquid more quantum is to lower the vitrification temperature (at least for the range of values of $h$ considered). 


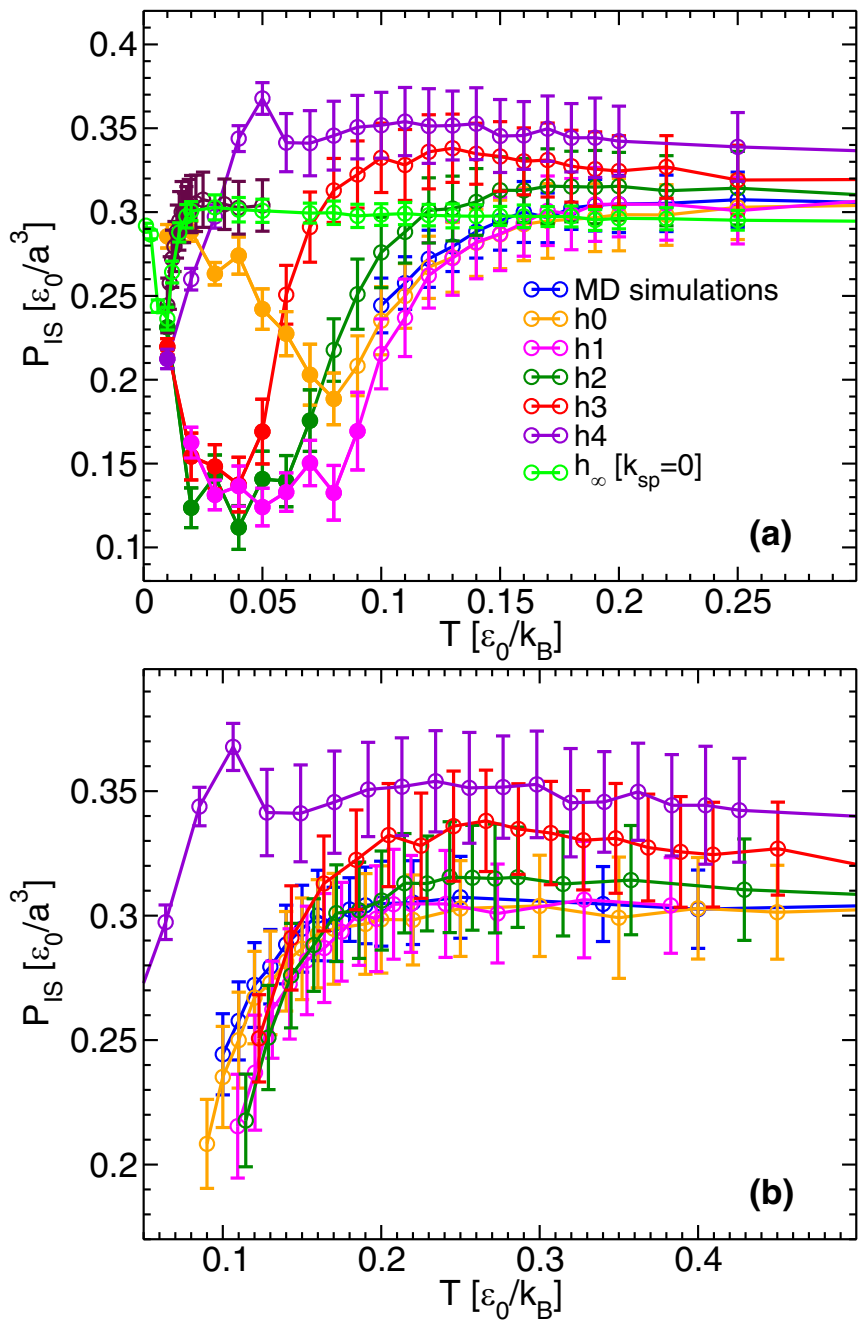

FIG. 3. (a) Inherent structure pressure as a function of temperature for the FJ liquids with $h_{0}=0$ (classical system), $h_{1}, h_{2}, h_{3}, h_{4}, h_{\infty}$. Empty and solid symbols correspond to equilibrium and out-of-equilibrium states, respectively. Also included are results obtained from MD simulations for the classical FJ liquid (blue line). The maroon line is obtained from the classical $P_{\mathrm{IS}}(T)$ ( $h_{0}=0$, orange line) by rescaling the temperature $T \rightarrow T / n_{b}$. (b) At approximately $T<0.15$, all $P_{\mathrm{IS}}(T)$ curves collapse within error bars onto the classical $P_{\mathrm{IS}}(T)$ if the temperature is rescaled as $T \rightarrow \gamma T$, where $\gamma(h)$ is obtained from Fig. 2(b) (Table I).

The nuclear quantum effects on $P_{\mathrm{IS}}(T)$ are shown in Fig. 3(a). Compared to Fig. 2, the fluctuations of $P_{\mathrm{IS}}(T)$ over the IS sampled by the system at a given $T$ are more relevant than in the case of $E_{\mathrm{IS}}(T)$. Nonetheless, it is apparent that the values of $P_{\mathrm{IS}}(T)$ for all the QFJ liquids studied converge to the classical IS pressures at high temperature, as expected. At low temperatures and in equilibrium (open circles), $P_{\mathrm{IS}}(T)$ increases with increasing $h$. However, this trend is reversed for $h>h_{4}$ since at low temperatures, $P_{\mathrm{IS}}(T)$ decreases as $h$ increases from $h=h_{4}$ to $h=h_{\infty}$. We also note that, as for the case of $E_{\mathrm{IS}}(T)$, the IS pressures obtained from our PIMC simulations for the case $h=h_{\infty}\left(k_{\mathrm{sp}}=0\right.$; green line $)$ are identical to the $P_{\mathrm{IS}}(T)$ of the classical FJ liquid after rescaling the temperature $T \rightarrow T / n_{b}$ (maroon line); see Sec. II of the SM [46].
Motivated by Fig. 2(b), we explore if the $P_{\mathrm{IS}}(T)$ of the different QFJ liquids also fall onto a master curve after rescaling the temperature. As shown in Fig. 3(b), the collapse of $P_{\mathrm{IS}}$ for different values of $h$ is limited to low temperatures, approximately $T<0.2$ within error bars. This is slightly different from Fig. 2(b), where the $E_{\mathrm{IS}}(T)$ of the different QFJ liquids collapse onto the classical values for all $T$ studied. We note that in Fig. 3(b), the temperatures are rescaled by $T \rightarrow \gamma T$, where $\gamma(h)$ is the same parameter used for $E_{\mathrm{IS}}(T)$ in Fig. 2(b); see Table I.

\section{B. Gaussian potential energy landscape: Configurational entropy}

A PEL is Gaussian if the corresponding distribution of IS energies $e_{\text {IS }}$ is given by

$$
\Omega\left(e_{\text {is }}\right)=\frac{1}{\sqrt{2 \pi} \sigma} \exp (\alpha N) \exp \left[-\left(e_{\text {is }}-E_{0}\right)^{2} /\left(2 \sigma^{2}\right)\right],
$$

where $\alpha, E_{0}$, and $\sigma$ depend only on the volume of the system $[37,38,61]$. Equation (1) implies that, for a given $N$ and $V$, most of the IS in the PEL have an energy $e_{\mathrm{IS}} \approx E_{0}$, while $\sigma$ quantifies the spread of the IS energies about $E_{0}$. The Gaussian approximation for $\Omega\left(e_{\text {is }}\right)$ is the basis for random energy models $[62,63]$ proposed for the study of disordered systems. In addition, numerical studies of so-called "fragile" liquids are consistent with Eq. (1) [27,64,65]. In particular, Eq. (1) is expected to hold for a system that can be considered to be composed of independent subsystems, each with its own independent IS energy [38].

As shown in Sec. III of the SM [46], in a Gaussian PEL,

$$
E_{\mathrm{IS}}(T)=E_{0}-\sigma^{2} /\left(k_{B} T\right)
$$

and

$$
P_{\mathrm{IS}}(T)=-\frac{d E_{0}}{d V}+N k_{B} T \frac{d \alpha}{d V}+\frac{1}{2 k_{B} T} \frac{d \sigma^{2}}{d V} .
$$

A natural question is whether these equations apply to the QFJ liquids studied, and hence if the corresponding PEL is Gaussian. We find that, remarkably, this is indeed the case at low temperatures, within the PEL-influenced regime. Figure 4(a) shows $E_{\mathrm{IS}}(T)$ and the best fit using Eq. (2) for all the QFJ liquids considered. The fitting parameters used in Eq. (2) are included in Table II. It follows from Table II that the effect of including quantum nuclear effects on the FJ liquid is to decrease $\sigma$ while maintaining $E_{0}$ practically constant. In other words, the distribution of IS energies $\Omega\left(e_{\text {is }}\right)$ becomes more narrow with increasing $h$, i.e., as the atoms become increasingly delocalized, while it remains centered at a fixed energy $e_{\text {is }}\left(\approx E_{0}\right)$. As shown in the inset of Fig. 4(a), $\sigma$ decreases linearly with increasing $h$ for $h \leqslant h_{3}$. This linear dependence seems to break down for $h>h_{3}$; we estimate that $\sigma^{2}=0.00015$ for $h=h_{4}$ and $n_{b}=20$ (see Sec. IV D).

The IS pressure of the QFJ liquids is shown in Fig. 4(b) together with the best fit using Eq. (3). Again, the fitting of $P_{\mathrm{IS}}(T)$ is limited to approximately $T<0.30$ because this is the temperature range corresponding to the PEL-influenced regime for all QFJ studied [see Fig. 2(a)]. The fitting parameters used in Eq. (3) are given in Table III. Interestingly, for all QFJ liquids, $d E_{0} / d V<0$ and $d \sigma^{2} / d V<0$, i.e., upon 

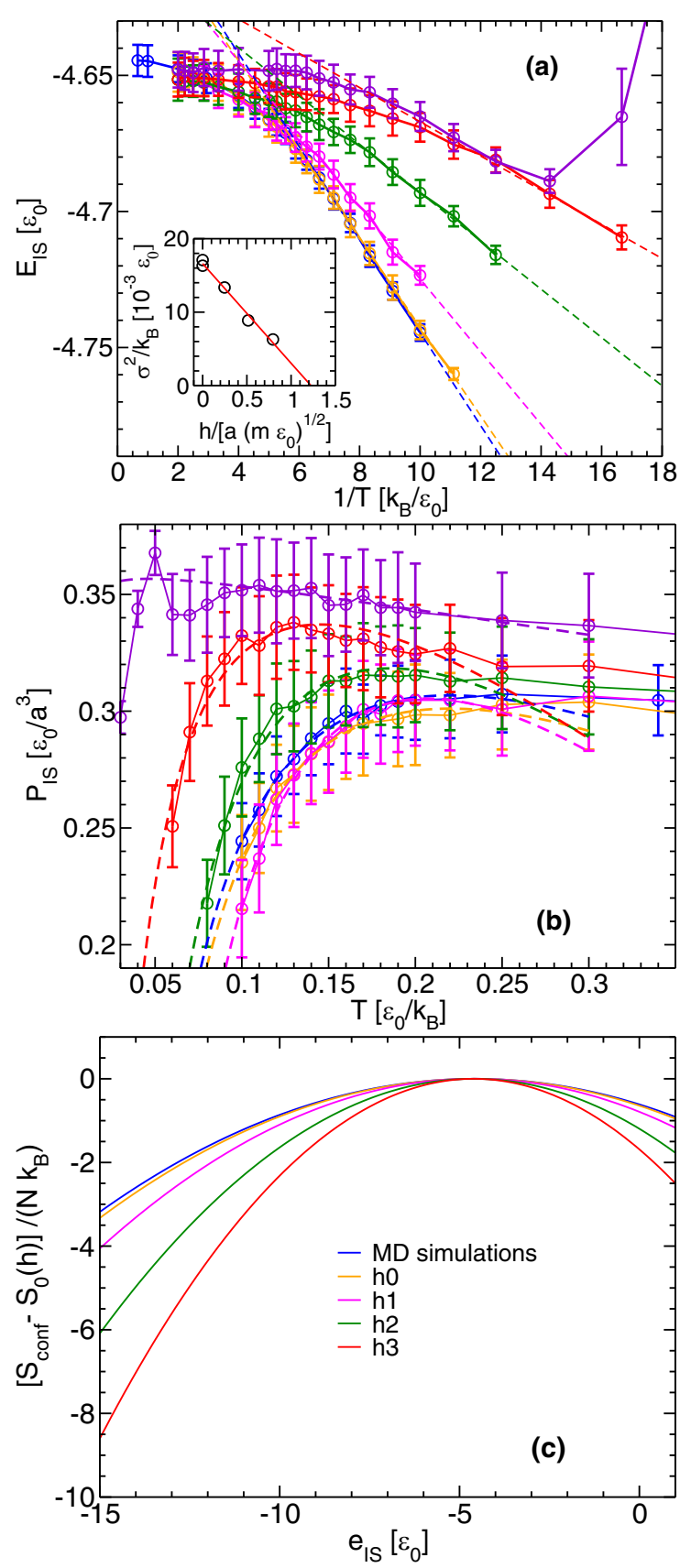

FIG. 4. (a) Inherent structure energies from Fig. 2(a) plotted as a function of $1 / T$. In the case of a Gaussian PEL, $E_{\mathrm{IS}}(T)=E_{0}-$ $\sigma^{2} /\left(k_{B} T\right)$ at low temperatures, where $E_{0}$ and $\sigma$ may depend only on $v$. Our results support that the PEL of the QFJ liquids studied is indeed Gaussian, at least for $h \leqslant h_{3}$, where sufficient equilibrium data are available (see the dashed lines). Inset: $\sigma^{2}$ as a function of $h$. (b) Similarly, in a Gaussian PEL, $P_{\mathrm{IS}}(T)=A+B T+C / T$, where $A, B, C$ are quantities that depend only on $v$ (see the text). The behavior of $P_{\mathrm{IS}}(T)$ at low temperatures also indicates that the PEL of the QFJ is Gaussian (see the dashed lines). (c) Configurational entropy of the QFJ liquids studied. Curves for different values of $h$ are shifted along the $y$-axis by an arbitrary constant $S_{0}(h)$. As the quantum nature of the liquids increases (i.e., $h$ increases), $S_{\text {conf }}\left(e_{\mathrm{IS}}\right)$ becomes thinner, but it remains centered at the same energy, $\approx-4.6$.
TABLE II. Fitting parameters for $E_{\mathrm{IS}}(T)$ in Fig. 4(a) based on Eq. (2), which holds for a Gaussian PEL.

\begin{tabular}{lcc}
\hline \hline$h\left[a\left(m \epsilon_{0}\right)^{1 / 2}\right]$ & $E_{0}\left(\epsilon_{0}\right)$ & $\sigma^{2} / k_{B}\left(\epsilon_{0}^{2} / k_{B}\right)$ \\
\hline Classical MD [50] & -4.5736 & 0.017087 \\
$h 0$ & -4.5793 & 0.016316 \\
$h 1$ & -4.5913 & 0.013353 \\
$h 2$ & -4.6043 & 0.008876 \\
$h 3$ & -4.6042 & 0.0062829 \\
\hline \hline
\end{tabular}

compression $(d V<0)$ about the volume $v=2.2$, the distribution of IS available in the PEL $\left[\Omega\left(e_{\text {is }}\right)\right]$ shifts toward larger energy values and becomes wider.

Since the PEL of the QFJ liquids studied are Gaussian $(h \leqslant$ $h_{4}$ ), one can easily compute the configurational entropy of these liquids, $S_{\text {conf }}=k_{B} \ln (\Omega)$ [38,64]. It follows from Eq. (1) that, for a Gaussian PEL, $S_{\text {conf }}$ is a quadratic function of $e_{\mathrm{IS}}$,

$S_{\text {conf }} / k_{B}=\alpha(V) N-\frac{\left[e_{\mathrm{IS}}-E_{0}(V)\right]^{2}}{2 \sigma^{2}(V)}-\ln [\sqrt{2 \pi} \sigma(V)]$,

or equivalently,

$$
S_{\text {conf }} / k_{B}=S_{0}(V, N)-\frac{\left[e_{\mathrm{IS}}-E_{0}(V)\right]^{2}}{2 \sigma^{2}(V)},
$$

where $S_{0}(V, N)=\alpha(V) N-\ln [\sqrt{2 \pi} \sigma(V)]$. Although all our simulations are performed at the same $(N, V)$, changing $h$ may affect $\alpha$, and hence Eq. (5) provides $S_{\text {conf }}$ for all QFJ liquids up to a function of $S_{0}=S_{0}(h)$. Figure $4(\mathrm{c})$ shows $S_{\text {conf }}\left(e_{\mathrm{IS}}\right)-$ $S_{0}(h)$ for all the QFJ liquids studied. It follows that, as the quantum nature of the liquid increases, $S_{\text {conf }}\left(e_{\mathrm{IS}}\right)$ becomes thinner and it remains centered at the same energy, $\approx-4.6$. The quantity $S_{0}(h)$, and hence $\alpha(h)$, are indeed important, but their calculation requires extensive additional work. These properties define the Kauzmann temperature $T_{K}(h)$ of the QFJ liquids, i.e., the temperature at which $S_{\text {conf }}=0$, below which the quantum system would get trapped in one IS (assuming the isomorphism between the classical ring-polymer system and the quantum liquid holds in this limit).

\section{Collapse of the ring-polymers at the IS}

In this section, we focus on the radius of gyration of the ring-polymers associated with the QFJ liquids. The radius of gyrations is calculated in both (i) the instantaneous configurations (obtained from the PIMC simulations) and (ii) the corresponding IS. Additional structural properties (bead-bead and centroid-centroid radial distribution functions) are included in Sec. IV of the SM [46].

TABLE III. Fitting parameters for $P_{\mathrm{IS}}(T)$ in Fig. 4(b) based on Eq. (3), which holds for a Gaussian PEL.

\begin{tabular}{lccc}
\hline \hline$h\left[a\left(m \epsilon_{0}\right)^{1 / 2}\right]$ & $-\frac{d E_{0}}{d V}\left[\epsilon_{0} / a^{3}\right]$ & $N k_{B} \frac{d \alpha}{d V}\left[k_{B} / a^{3}\right]$ & $\frac{1}{2 k_{B}} \frac{d \sigma^{2}}{d V}\left[\epsilon_{0}^{2} / a^{3} k_{B}\right]$ \\
\hline MD simulations & 0.49627 & -0.43046 & -0.0208576 \\
$h_{0}$ & 0.498387 & -0.448131 & -0.0217122 \\
$h_{1}$ & 0.626957 & -0.778775 & -0.0331937 \\
$h_{2}$ & 0.566953 & -0.670497 & -0.0230476 \\
$h_{3}$ & 0.515117 & -0.612217 & -0.0129385 \\
\hline \hline
\end{tabular}



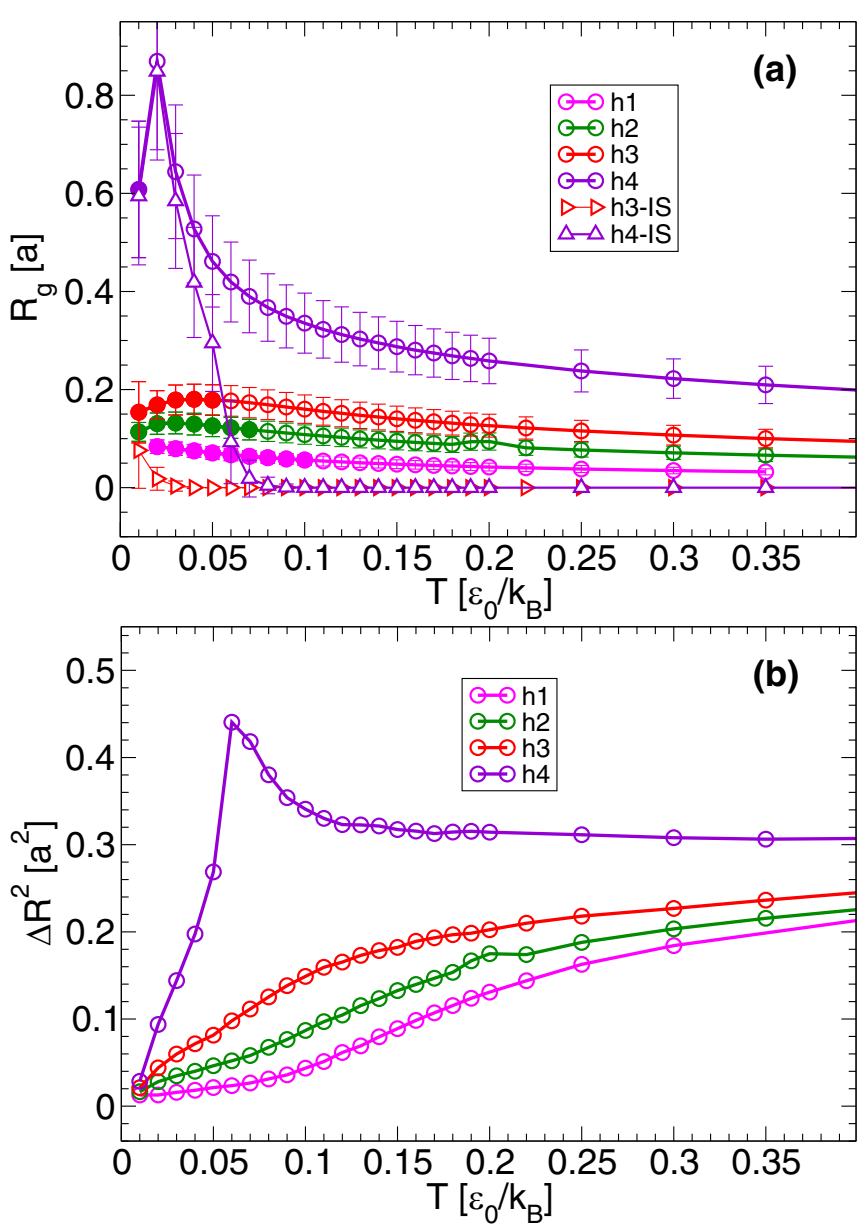

FIG. 5. (a) Radius of gyration $R_{g}$ of the ring-polymers associated with the QFJ liquids as a function of temperature (circles; empty and solid symbols correspond to equilibrium and out-of-equilibrium states, respectively). As the system becomes more quantum ( $h$ increases), $R_{g}(T)$ increases, indicating that the atoms in the QFJ liquids become more delocalized (as expected). Also included is the radius of gyration of the ring-polymers at the IS, $R_{g, \mathrm{IS}}(T)$, for the cases $h=h_{3}$ and $h_{4}$ (triangles). For $h \leqslant h_{3}, R_{g, \text { IS }}(T) \approx 0$ for all $T$, i.e., the ring-polymers at the IS are collapsed; see, e.g., red triangles for $h=$ $h_{3}$. For $h=h_{4}, R_{g, \mathrm{IS}}(T) \approx 0$ at $T>0.07$, while at $T<0.07, R_{g, \mathrm{IS}}(T)$ grows rapidly (i.e., the ring-polymers at the IS do not collapse). However, our results for $h=h_{4}$ are reliable only at $T>0.07$ (see Sec. IV D). (b) Average square distance in configuration space, $\Delta R^{2}$, between the instantaneous configurations sampled by the system and the corresponding IS. At all temperatures, the IS and instantaneous configurations of the QFJ liquids are different, $\Delta R^{2}>0$, even for the cases in which $R_{g}(T) \approx R_{g, \mathrm{IS}}(T) \approx 0.06-0.08\left(h=h_{4}\right.$ and $T \leqslant 0.03$ ).

We discuss first the average radius of gyration $R_{g}(T)$ of the ring-polymers. $R_{g}(T)$ quantifies how spread the beads of the ring-polymers are from the corresponding center of mass. From the quantum mechanics perspective, $R_{g}^{2}(T)$ is the (quantum) uncertainty in the position of the atoms of the QFJ liquid. As shown in Fig. 5(a), in equilibrium (open circles), $R_{g}(T)$ is a monotonic decreasing function of $T$ for all the QFJ liquids, indicating that the atoms in the liquid become more localized with increasing $T$ (i.e., as the classical limit is approached). This is expected since the spring constant of the ring-polymers increases with increasing $T$. Figure 5(a) also shows the effects of increasing the quantumness of the QFJ liquids (i.e., $h$ ) at a given $T$. For $h \leqslant h_{3}$, and for the equilibrated states (open circles), $R_{g}(T)$ increases with increasing $h$, as expected. After all, increasing $h$ at a constant temperature reduces the spring constant of the ring-polymers, making the ring-polymers/atoms of the QFJ liquid increasingly delocalized. The same trend with $T$ and $h$ holds if we include the case $h=h_{4}$ for $T \geqslant 0.07$. Again, these are the temperatures at which results for $h=h_{4}$ are insensitive to $n_{b}$, and hence reliable; the results obtained at $T \leqslant 0.07$ for $h=h_{4}$ will be discussed in Sec. IV D. We note that, for $h \leqslant h_{3}$ in the equilibrium regime (open circles) and for $h=h_{4}$ at $T>0.07, R_{g}(T)<0.4$, i.e., the atoms in the QFJ liquids are somewhat delocalized with the associated beads spreading for up to $\approx 40 \%$ the hard-core distance of the FJ pair potential.

Perhaps the most surprising result regarding the ringpolymers structure is their average radius of gyration at the $I S, R_{g, \mathrm{IS}}(T)$. We find that, for $h \leqslant h_{3}$ and in the equilibrium regime, the ring-polymers are collapsed at the IS. As an example, included in Fig. 5(a) is $R_{g, \text { IS }}(T)$ for $h_{3}$ (similar results hold for $h=h_{1}, h_{2}$ ). In this case, $R_{g, \text { IS }}(T)=0$ for $T \geqslant 0.06$, and only at very low temperature, in the out-of-equilibrium domain (solid circles), does $R_{g, \mathrm{IS}}(T)$ become non-negligible. Still, even at these very low temperatures, $R_{g, \text { IS }}<R_{g}$, i.e., the ring-polymers partially contract at the IS, i.e., during the potential energy minimization.

The case $h=h_{4}$ deserves some clarification. Again, at this level of quantumness, our results are $n_{b}$-independent only for $T \geqslant 0.07$. At these temperatures, we also find that $R_{g, \text { IS }}(T)=0$, as found for $h \leqslant h_{3}$; see the violet triangles in Fig. 5(a). At $T<0.07$, the $R_{g, \mathrm{IS}}(T)$ increases rapidly upon cooling, and at $T \leqslant 0.02$ it is found that $R_{g}(T)=R_{g, \mathrm{IS}}(T) \approx$ $0.6-0.8$. At these conditions, the ring-polymers in the instantaneous configurations and in the IS are considerably spread. However, as we will show in the next section, the sudden increase in $R_{g, \mathrm{IS}}$ at $T<0.07$ [which is also correlated to the sudden increase in $E_{\mathrm{IS}}(T)$ in Fig. 2] shifts to lower $T$ with increasing $n_{b}$. This suggests that for our atomistic systems, $R_{g, \text { IS }}(T)=0$ also for $h=h_{4}$. We note that even in the case in which $R_{g}(T)=R_{g, \mathrm{IS}}(T)\left(T=0.01-0.02\right.$ and $\left.h=h_{4}\right)$, the instantaneous and IS configurations are different. To show this, we include in Fig. 5(b) the square of the distance (in the $\left[\left(3 \mathrm{Nn} n_{b}\right)\right.$-dimensional] configurational space of the ringpolymer system) between (i) the instantaneous configuration and (ii) the corresponding IS in configurational space $\Delta R^{2}$ normalized by the number of beads in the system,

$$
\Delta R^{2} \equiv \frac{1}{N n_{b}} \sum_{i, k=1}^{i=N ; k=n_{b}}\left(\vec{r}_{i, k}-\vec{r}_{i, k}^{\mathrm{IS}}\right)^{2} .
$$

Here, $\vec{r}_{i, k}$ and $\vec{r}_{i, k}^{I S}$ are, respectively, the position of bead $k$ of ring-polymer $i$ in the instantaneous configuration, and the IS of the ring-polymer system. Equation (6) can also be interpreted as the average distance in real (three-dimensional) space between the position of the beads in the (i) instantaneous configuration and (ii) corresponding IS. It follows from Fig. 5(b) that $\Delta R>0$ at all temperatures and for all $h \leqslant h_{4}$, and hence the instantaneous and IS of the quantum/ringpolymer systems are indeed different. 


\section{Effects of varying the beads number on the PEL properties}

Strictly speaking, in the path integral formulation of statistical mechanics, one must take $n_{b} \rightarrow \infty$. In computer simulations, one usually considers a "large" value of $n_{b}$, so results from computer simulations do not change upon further increasing $n_{b}$. In this section, we explore whether (and how) our results vary with $n_{b}$ for all degrees of quantumness (i.e., values of $h$ ) explored.

As shown in Sec. V of the SM [46], $n_{b}$ has minor or no effects on our results obtained for $h \leqslant h_{3}$. However, for $h=h_{4}$, the properties of the QFJ liquid change with $n_{b}$ at low temperatures. Figures $6(\mathrm{a})$ and $6(\mathrm{~b})$ show $E_{\mathrm{IS}}(T)$ and $P_{\mathrm{IS}}(T)$ for the QFJ liquid with $h=h_{4}$ and for PIMC simulations performed with $n_{b}=5,10,15,20$ beads per ring-polymer. At very low temperatures, $E_{\mathrm{IS}}(T)$ develops a very large anomalous maximum, not observed at $h \leqslant h_{3}$. The maxima in $E_{\mathrm{IS}}(T)$ are accompanied by a small maximum in $P_{\mathrm{IS}}(T)$. The main point of Figs. 6(a) and 6(b) is that $E_{\mathrm{IS}}(T)$ and $P_{\mathrm{IS}}(T)$ are $n_{b}$-independent only at high temperatures. For example, the values of $E_{\mathrm{IS}}(T)$ and $P_{\mathrm{IS}}(T)$ obtained for $n_{b}=5$ are reliable only down to $T^{*}=0.09$, while for $n_{b}=10,15,20$ this temperature shifts to $T^{*}=0.07,0.06,0.05$, respectively. It is apparent that increasing $n_{b} \rightarrow \infty$ would remove (or shift to $T \rightarrow 0$ ) the anomalous maxima in $E_{\mathrm{IS}}(T)$ and $P_{\mathrm{IS}}(T)$, and hence, in this limit, the results obtained for the QFJ liquid at $h=h_{4}$ would be qualitatively similar to those obtained at $h \leqslant h_{3}$. We note that the conclusions from Fig. 6 are not affected by the lack of equilibration, since increasing $n_{b}$ allows the system to relax to equilibrium increasingly faster. For example, as shown in Sec. V of the SM [46], the lowest temperature at which the system reaches equilibrium for the case $h=h_{4}$ is $T=0.02$ for $n_{b}=10$, while for $n_{b}=20$ the system is in equilibrium at all temperatures studied, $T \geqslant 0.01$.

A similar conclusion follows when one looks at $R_{g, \mathrm{IS}}(T)$ for the QFJ with $h=h_{4}$. As shown in Fig. 7, at $T>T^{*}(h)$, $R_{g, \text { IS }}(T)=0$, independently of $n_{b}$. Hence, as found for $h \leqslant$ $h_{3}$, the ring-polymers are collapsed at the IS. The (anomalous) sharp increase in $R_{g, \text { IS }}(T)$ at $T<T^{*}(h)$ is also strongly dependent on $n_{b}$. As for the case of $E_{\mathrm{IS}}(T)$ and $P_{\mathrm{IS}}(T)$, the anomalous increase in $R_{g, \mathrm{IS}}(T)$ is apparently suppressed in the limit $n_{b} \rightarrow \infty$. We performed simulations with an even larger Planck constant, $h_{4 b}=3.5764>h_{4}$, and we found results similar to those presented in Figs. 6 and 7 for $h=h_{4}$. The corresponding results for $h=h_{4 b}$ as well as $h \leqslant h_{3}$ are included in the SM [46].

\section{E. A description of quantum liquids using the PEL of the corresponding classical liquid}

Since the ring-polymers collapse during the potential energy minimization, it follows that the IS obtained from the equilibrium quantum liquids $\left(h \leqslant h_{4}\right)$ define a unique configuration of the classical liquid. It also follows that such a classical configuration must be an IS of the classical liquid. In other words, an IS of the quantum liquid, in the ring-polymer system PEL, is also an IS of the classical liquid PEL. This IS is defined by the $3 N$ coordinates of the ring-polymer beads belonging to any of the $n_{b}$ replicas of the system (since the ring-polymers collapse at the IS, all replicas are defined by the same set of $3 N$ bead coordinates). One may wonder, how
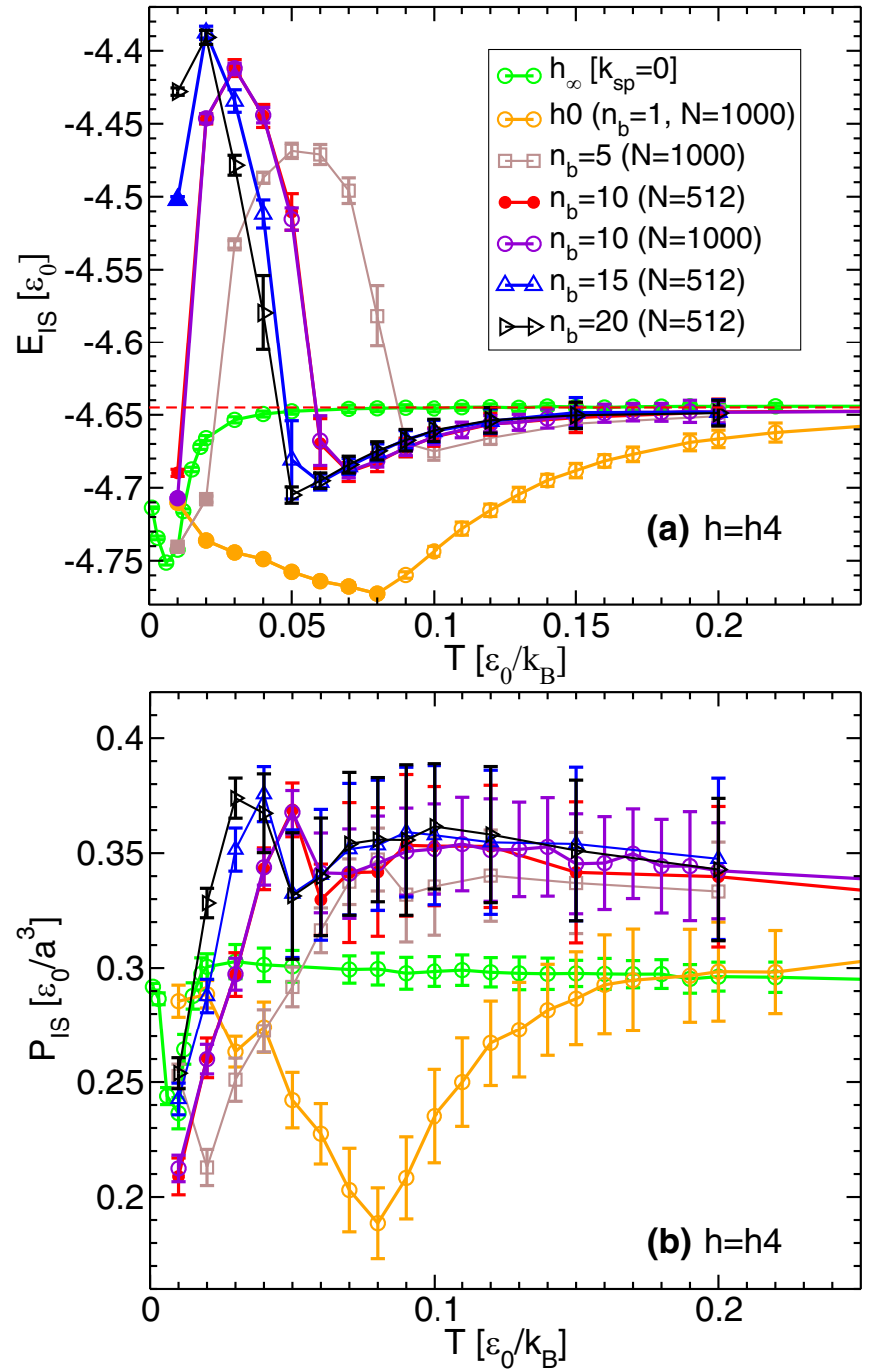

FIG. 6. Average IS (a) energy and (b) pressure as function of temperature for the QFJ liquid with Planck's constant $h=h_{4}$ and simulated using different numbers of beads per ring-polymer $n_{b}$. The number of ring-polymers (atoms) in the system is $N=512$ for $n_{b}=$ 15,20 , and $N=1000$ for $n_{b}=1,5,10$. Results for $\left(n_{b}=10, N=\right.$ $1000)$ (violet) and ( $\left.n_{b}=10, N=512\right)$ (red) are practically identical, suggesting that there are no finite-size effects. For comparison, also shown are the results for $h=h_{0}$ (classical case) and $h=h_{\infty}$ (spring constant of the ring-polymers equal to zero). Empty (solid) symbols in (a) indicate temperatures at which the system reaches (does not reach) equilibrium. Increasing $n_{b}$ shifts the (anomalous) maximum in $E_{\mathrm{IS}}(T)$ and the low- $T$ maximum in $P_{\mathrm{IS}}(T)$ toward lower $T$.

do the IS of the QFJ liquids, in the CL-PEL, compare with the IS sampled by the classical liquid in equilibrium (in the CL-PEL)? Specifically, are the IS of the quantum liquids also sampled by the classical liquid? Or, are there novel IS accessible to the quantum liquid that, e.g., are never sampled by the classical liquid? The collapse of the $E_{\mathrm{IS}}(T)$ and $P_{\mathrm{IS}}(T)$ curves of the QFJ liquids onto the classical liquid $E_{\mathrm{IS}}(T)$ and $P_{\mathrm{IS}}(T)$ curves [Figs. 2(b) and 3(b)] strongly suggests that the quantum and classical liquids explore similar, if not the same, IS of the CL-PEL.

As discussed in the previous sections, in the case of the nonequilibrium QFJ liquids with $h \leqslant h_{3}$ and at $T \leqslant 0.07$ for 


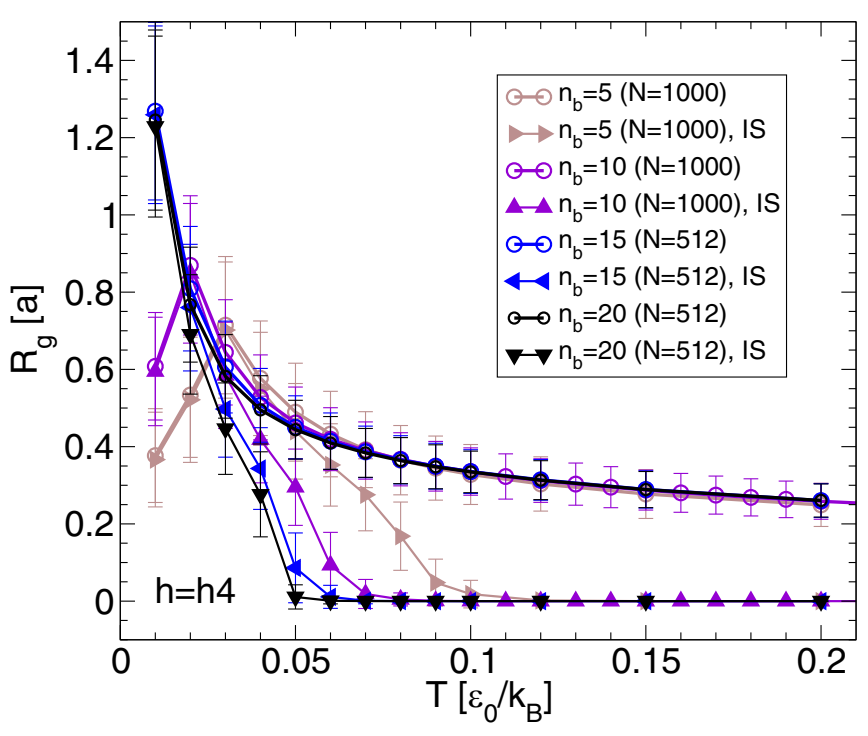

FIG. 7. Radius of gyration of the ring-polymers $R_{g}$ associated with the QFJ liquids at the same conditions as in Fig. 6 (circles). For comparison, we include the radius of gyration of the ring-polymers at the corresponding IS (triangles). Increasing $n_{b}$ shifts the (anomalous) maxima in $R_{g, \mathrm{IS}}(T)$ toward lower $T$.

the case $h=h_{4}$, potential energy minimization of the quantum liquid does not necessarily lead to the collapse of the ringpolymers [Fig. 5(a)]. In these cases, an IS of the quantum liquid does not define an IS of the CL-PEL. Instead, after the potential energy is minimized, each of the $n_{b}$ replicas associated with the quantum liquid defines a distinct point of the CL-PEL, which may or may not correspond to an IS of the CL-PEL.

In general, the quantum liquid/ring-polymer system is represented by a set of $n_{b}$ points in the CL-PEL [Fig. 1(b)] and, in the limit $n_{b} \rightarrow \infty$, this set of points would form a pancakelike patch that spreads over the CL-PEL [Fig. 1(c)]. As time evolves, this patch changes shape and moves, describing a fuzzy trajectory on the CL-PEL. Interestingly, we find that at all temperatures studied, such a representative "patch" of the quantum liquid $\left(h \leqslant h_{4}\right)$ in the CL-PEL is distributed over different neighboring basins of the CL-PEL. To show this, we perform the following analysis (at a given $h$ and $T$ ). (i) First, we select $N_{c}=10$ instantaneous configurations of the target quantum liquid. From each of these configurations, we obtain the corresponding $n_{b}$ replicas; these replicas define $n_{b}$ points in the CL-PEL. (ii) Each of these $n_{b}$ replicas is treated as an independent classical liquid configuration, and it is subjected to potential energy minimization. As a result, we obtain $n_{b}$ inherent structures of the CL-PEL. (iii) Finally, we calculate the distance square in configurational space $d_{k, k+1}^{2}$ (normalized by $N$ ) between the IS of the CL-PEL obtained from replicas $k$ and $k^{\prime}=k+1\left(\right.$ where $\left.k=1,2, \ldots, n_{b}\right)$,

$$
d_{k, k+1}^{2}=1 / N \sum_{i=1}^{i=N}\left(\vec{r}_{i, k}-\vec{r}_{i, k+1}\right)^{2},
$$

where $\vec{r}_{i, k}$ is the position of "particle" $i=1,2, \ldots, N$ of replica $k$ after potential energy minimization, and $\vec{r}_{i, n_{b}+1} \equiv$

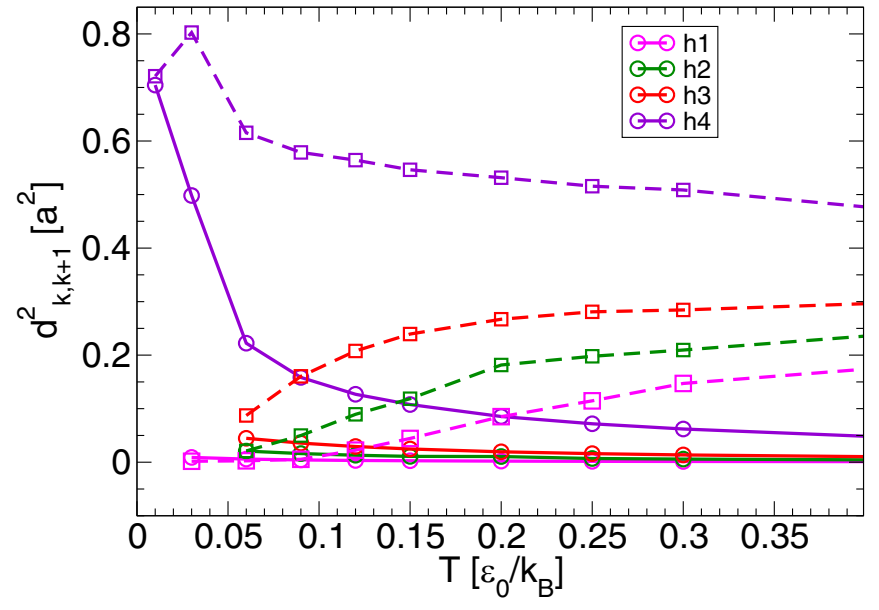

FIG. 8. Average distance between replicas $k$ and $k+1$ in the CL-PEL as function of temperature, $d_{k, k+1}(T)$. A ring-polymer configuration associated with the quantum liquid at a given $(T, h)$ defines $n_{b}$ replicas $\left(k=1,2, \ldots, n_{b}\right)$. These replicas define $n_{b}$ independent points (i.e., classical liquid configurations) on the CL-PEL. The solid lines represent $d_{k, k+1}(T)$ from the replicas obtained directly from the PIMC simulation; dashed lines represent $d_{k, k+1}(T)$ after the individual replicas are subjected to potential-energy minimization (in the CL-PEL).

$\vec{r}_{i, 1}$. If the $n_{b}$-replicas are in the same basin of the CL-PEL, then they should converge to the same IS (of the CL-PEL) after each of them is subjected to potential energy minimization, i.e., $d_{k, k+1}=0$ for all $k$. Instead, as shown in Fig. 8, we find that $d_{k, k+1}>0$ (dashed lines), indicating that the replicas of a given quantum liquid/ring-polymer configuration occupy different IS of the CL-PEL. Figure 8 also includes the values of $d_{k, k+1}$ obtained from the instantaneous configurations of the replicas, before the minimization of the potential energy (solid lines). Interestingly, we find that $d_{k, k+1}$ increases after potential energy minimization. This means that the replicas spread even further on the CL-PEL after they reach the corresponding IS of the CL-PEL. It follows that the collapse of the ring-polymers at the IS of the PEL must be due to the springs that keep the bead polymers together. This is because, without the springs, the beads of the ring-polymers do not overlap after potential energy minimization $\left(d_{k, k+1}>0\right)$.

\section{SUMMARY AND DISCUSSION}

In this work, we extended the PEL formalism, originally proposed to study classical liquids and glasses, to the case of quantum liquids. This was done by exploiting the isomorphism between (i) quantum liquids and (ii) classical ring-polymer systems that follows from the path-integral statistical mechanics formulation for quantum systems [44]. In the case of an atomistic classical/quantum liquid, the system is represented by a single point in the corresponding PEL; see Fig. 1(a). In the classical case, the PEL resides in a $(3 N+1)$-dimensional space where the generalized coordinates $q$ [axes in Fig. 1(a)] are the atoms' coordinates. In the case of the quantum liquid, the PEL is defined by the isomorphic ring-polymer system. If the ring-polymers are composed of $n_{b}$ beads each, then the (quantum liquid) PEL 
resides in a $\left(3 N n_{b}+1\right)$-dimensional space where the generalized coordinates $q$ [axes in Fig. 1(a)] are the coordinates of the ring-polymers beads. It follows that the same picture used extensively to describe the behavior of classical liquids can be extended to quantum liquids. Specifically, at high temperatures, the representative point of the quantum/classical liquid is able to explore wide regions of the PEL (PEL-independent regime). As temperature decreases, the representative point of the system is unable to overcome large potential energy barriers, and hence the system is able to explore increasingly limited regions of the PEL. The isomorphism between quantum liquids and ring-polymer systems holds only in equilibrium. Yet, it is tempting to extrapolate these ideas to the out-of-equilibrium glass state. If so, the liquid-to-glass transition of the quantum liquid, within the PEL formalism, would not be different from the classical case. Specifically, at very low temperatures, in the glass state, the representative point of the quantum/classical liquid is trapped within one basin of the PEL, and only vibrational motion is possible. The ideas presented above are relatively easy to visualize when $n_{b}$ is finite, as is always the case with PIMD/PIMC computer simulations. We note, however, that in theory, $n_{b} \rightarrow \infty$ implying that the PEL of a quantum liquid is indeed a hypersurface in a $\left(3 N n_{b}+1\right) \rightarrow \infty$-dimensional space.

Our PIMC simulations indicate that, in the PELindependent regime, $E_{\mathrm{IS}}(T)$ and $P_{\mathrm{IS}}(T)$ are constant and hence independent of the topography of the PEL. Instead, in the PEL-influenced regime, $E_{\mathrm{IS}}(T)$ and $P_{\mathrm{IS}}(T)$ vary with $T$. The crossover temperature separating the PEL-independent and PEL-influenced regimes is well-defined. The effect of increasing the quantumness of the liquid, i.e., increasing the value of Planck's constant, is to lower this crossover temperature. This means that, at the conditions explored, the glass transition temperature of the liquid decreases as the atoms become more delocalized.

The values of $h$ considered correspond to quantum liquids that exhibit relevant quantum behavior. Specifically, the de Broglie thermal wavelength of these liquids at $T=0.10$ is comparable to the de Broglie thermal wavelength of $\mathrm{H}_{2}$ at $T=$ $10-300 \mathrm{~K}$. Indeed, we observed that atoms in our computer simulation can be mildly delocalized. Depending on temperature, the beads of the ring-polymer system can be as far as $\approx 40 \%$ of the hard-core radius from the corresponding ringpolymer center of mass. In the context of the PEL, this means that the representative point of the system is always defined by ring-polymers that are spread in real (three-dimensional) space. Remarkably, we also find that during potential-energy minimization, all the ring-polymers of the system collapse. Accordingly, when the representative point of the system is at an IS of the PEL, all ring-polymers are effectively point particles. Thus, the quantum configuration of the system becomes a classical liquid configuration (defined by $3 N$-coordinates); see below.

Our simulations are based on the Fermi-Jagla model liquid. In this case, the classical liquid was found to be characterized by a Gaussian PEL. In particular, it was found that increasing $h$, i.e., making the liquid increasingly quantum, does not affect the nature of the PEL, i.e., all quantum liquids studied are also characterized by a Gaussian PEL. The PEL of the quantum and classical liquids are all centered at the same IS energy $E_{0}$.
The effect of increasing $h$ is to make the distribution of IS energies narrower (i.e., all IS structures of the PEL become closer to $E_{0}$ ). These conclusions have a direct impact on the configurational entropy of the system; see Fig. 4.

The extension of the PEL approach to quantum systems provides a tool to understand the behavior of low-temperature quantum liquids at low temperatures and the liquid-to-glass transition. From a more applied point of view, the PEL formalism can be used to obtain the Helmholtz free energy of the system $F(N, V, T)$ in terms of PEL properties, such as $S_{\text {conf }}$ and $E_{\mathrm{IS}}$. This is particularly useful in the context of computer simulations at temperatures where the required timescales are not accessible, the case of computational studies of supercooled water being a relevant example [66]. Calculations of $F(N, V, T)$ at very low temperatures using the PEL approach are possible if the PEL of the system of interest is harmonic and Gaussian. Hence, it is particularly encouraging that the PEL of all our quantum liquids are indeed Gaussian.

The PEL approach described above for quantum liquids is a literal application of the PEL formalism for classical liquids. Specifically, both quantum and classical liquids can be described by a representative point moving on a PEL, as shown in Fig. 1(a). In this work, we also discussed an alternative PEL-based description of the quantum liquid using the classical liquid PEL (CL-PEL), as opposed to the PEL of the associated ring-polymer system. The basic idea is summarized in Figs. 1(b) and 1(c). Briefly, if the ring-polymers are composed of $n_{b}$-beads, the system is represented by $n_{b}$ points in the CL-PEL. As time evolves, the $n_{b}$-points representing the system (replicas) move, describing $n_{b}$ trajectories on the PEL [Fig. 1(b)]. In the limit $n_{b} \rightarrow \infty$, the $n_{b}$-points in the CLPEL representing the quantum liquid become, collectively, a pancakelike patch [Fig. 1(c)]. The representative patch of the system travels with time, changing shape, and tracing a fuzzy trajectory on the CL-PEL. It follows that, at high temperatures, the quantum liquid is able to explore wide regions of the PEL (PEL-independent regime). As temperature decreases, the representative patch of the system is unable to overcome large potential energy barriers, and hence the system is able to explore limited regions of the PEL. At the lowest temperatures studied, we find that the representative patch of the quantum liquids spreads over a few IS of the CL-PEL. We note that one advantage of the CL-PEL relative to the ring-polymer system PEL is that the number of generalized coordinates in the CL-PEL is finite, $3 N$. Instead, the number of dimensions of the ring-polymer system PEL $\rightarrow \infty$ since $n_{b} \rightarrow \infty$. In addition, while the CL-PEL is temperature-independent, the ring-polymer PEL is not. This may make the description of the quantum liquid based on the CL-PEL more intuitive.

\section{ACKNOWLEDGMENTS}

We acknowledge support from the NSF CREST Center for Interface Design and Engineered Assembly of Low Dimensional systems (IDEALS), NSF Grant No. HRD-1547830. This work was also supported, in part, by a grant of computer time from the City University of New York High Performance Computing Center under NSF Grants No. CNS-0855217, No. CNS-0958379, and No. ALI-1126113. 
[1] C. A. Angell, Formation of glasses from liquids and biopolymers, Science 267, 1924 (1995).

[2] P. G. Debenedetti and F. H. Stillinger, Supercooled liquids and the glass transition, Nature (London) 410, 259 (2001).

[3] W. Kauzmann, The nature of the glassy state and the behavior of liquids at low temperatures, Chem. Rev. 43, 219 (1948).

[4] P. G. Debenedetti, Metastable Liquids: Concepts and Principles (Princeton University Press, Princeton, NJ, 1996).

[5] K. Binder and W. Kob, Glassy Materials and Disordered Solids (World Scientific, Singapore, 2005).

[6] W. Götze, Recent tests of the mode-coupling theory for glassy dynamics, J. Phys. Condens. Matter 11, A1 (1999).

[7] A. Widmer-Cooper and P. Harrowell, Predicting the Long-Time Dynamic Heterogeneity in a Supercooled Liquid on the Basis of Short-Time Heterogeneities, Phys. Rev. Lett. 96, 185701 (2006).

[8] V. Lubchenko and P. G. Wolynes, Theory of structural glasses and supercooled liquids, Annu. Rev. Phys. Chem. 58, 235 (2007).

[9] J. C. Dyre, The glass transition and elastic models of glassforming liquids, Rev. Mod. Phys. 78, 953 (2006).

[10] W. Götze, Complex Dynamics of Glass-Forming Liquids: A Mode-Coupling Theory (Oxford University Press, Oxford, 2008).

[11] M. D. Ediger and P. Harrowell, Supercooled liquids and glasses, J. Chem. Phys. 137, 080901 (2012).

[12] L. Berthier and G. Biroli, Theoretical perspective on the glass transition and amorphous materials, Rev. Mod. Phys. 83, 587 (2011).

[13] H. Tong and H. Tanaka, Structural order as a genuine control parameter of dynamics in simple glass formers, Nat. Commun. 10, 5596 (2019).

[14] M. A. Morales, C. Pierleoni, E. Schwegler, and D. M. Ceperley, Evidence for a first-order liquid-liquid transition in highpressure hydrogen from $a b$ initio simulations, Proc. Natl. Acad. Sci. USA 107, 12799 (2010).

[15] M. Boninsegni, N. Prokof'ev, and B. Svistunov, Superglass Phase of ${ }^{4} \mathrm{He}$, Phys. Rev. Lett. 96, 105301 (2006).

[16] M. Ceriotti, W. Fang, P. G. Kusalik, R. H. McKenzie, A. Michaelides, M. A. Morales, and T. E. Markland, Nuclear quantum effects in water and aqueous systems: Experiment, theory, and current challenges, Chem. Rev. 116, 7529 (2016).

[17] C. Gainaru, A. L. Agapov, V. Fuentes-Landete, K. AmannWinkel, H. Nelson, K. W. Köster, A. I. Kolesnikov, V. N. Novikov, R. Richert, R. Böhmer, T. Loerting, and A. P. Sokolov, Anomalously large isotope effect in the glass transition of water, Proc. Natl. Acad. Sci. USA 111, 17402 (2014).

[18] K. H. Kim, A. Späh, H. Pathak, F. Perakis, D. Mariedahl, K. Amann-Winkel, J. A. Sellberg, J. H. Lee, S. Kim, J. Park et al., Maxima in the thermodynamic response and correlation functions of deeply supercooled water, Science 358, 1589 (2017).

[19] A. Amir, Y. Oreg, and Y. Imry, Slow Relaxations and Aging in the Electron Glass, Phys. Rev. Lett. 103, 126403 (2009).

[20] M. H. Wu, B. Ellman, T. F. Rosenbaum, G. Aeppli, and D. H. Reich, From Classical to Quantum Glass, Phys. Rev. Lett. 67, 2076 (1991).

[21] G. Biroli, C. Chamon, and F. Zamponi, Theory of the superglass phase, Phys. Rev. B 78, 224306 (2008).
[22] B. Hunt, E. Pratt, V. Gadagkar, M. Yamashita, A. V. Balatsky, and J. C. Davis, Evidence for a superglass state in solid ${ }^{4} \mathrm{He}$, Science 324, 632 (2009).

[23] R. A. Klemm, Quantum effects in spin glasses, J. Phys. C 12, L735 (1979).

[24] T. Markland, J. A. Morrone, B. J. Berne, K. Miyazaki, E. Rabani, and D. R. Reichman, Quantum fluctuations can promote or inhibit glass formation, Nat. Phys. 7, 134 (2011).

[25] T. Yokota, Infinite range ising spin glass model with a transverse field, Phys. Lett. A 125, 482 (1987).

[26] T. E. Markland, J. A. Morrone, K. Miyazaki, B. J. Berne, D. R. Reichman, and E. Rabani, Theory and simulations of quantum glass forming liquids, J. Chem. Phys. 136, 074511 (2012).

[27] F. W. Starr, S. Sastry, E. La Nave, A. Scala, H. E. Stanley, and F. Sciortino, Thermodynamic and structural aspects of the potential energy surface of simulated water, Phys. Rev. E 63, 041201 (2001).

[28] I. Saika-Voivod, F. Sciortino, and P. H. Poole, Free energy and configurational entropy of liquid silica: Fragile-to-strong crossover and polyamorphism, Phys. Rev. E 69, 041503 (2004).

[29] P. H. Handle, F. Sciortino, and N. Giovambattista, Glass polymorphism in TIP4P/2005 water: A description based on the potential energy landscape formalism, J. Chem. Phys. 150, 244506 (2019).

[30] J. Engstler and N. Giovambattista, Heating- and pressureinduced transformations in amorphous and hexagonal ice: A computer simulation study using the TIP4P/2005 model, J. Chem. Phys. 147, 074505 (2017).

[31] J. Wong, D. A. Jahn, and N. Giovambattista, Pressure-induced transformations in glassy water: A computer simulation study using the TIP4P/2005 model, J. Chem. Phys. 143, 074501 (2015).

[32] N. Giovambattista, F. W. Starr, and P. H. Poole, State variables for glasses: The case of amorphous ice, J. Chem. Phys. 150, 224502 (2019).

[33] D. J. Wales, Energy Landscapes (Cambridge University Press, Cambridge, UK, 2003).

[34] S. Sastry, P. G. Debenedetti, and F. H. Stillinger, Statistical geometry of particle packings. II. "Weak Spots" in liquids, Phys. Rev. E 56, 5533 (1997).

[35] F. Sciortino and P. Tartaglia, Extension of the FluctuationDissipation Theorem to the Physical Aging of a Model Glass-Forming Liquid, Phys. Rev. Lett. 86, 107 (2001).

[36] M. S. Shell, P. G. Debenedetti, F. Sciortino, and E. La Nave, Energy landscapes, ideal glasses, and their equation of state, J. Chem. Phys. 118, 8821 (2003).

[37] F. H. Stillinger, Energy Landscapes, Inherent Structures, and Condensed-Matter Phenomena (Princeton University Press, Princeton, NJ, 2016).

[38] F. Sciortino, Potential energy landscape description of supercooled liquids and glasses, J. Stat. Mech. (2005) P05015.

[39] M. Goldstein, Viscous liquids and the glass transition: A potential energy barrier picture, J. Chem. Phys. 51, 3728 (1969).

[40] M. Goldstein, Viscous liquids and the glass transition. VII. molecular mechanisms for a thermodynamic second order transition, J. Chem. Phys. 67, 2246 (1977)

[41] F. H. Stillinger and T. A. Weber, Hidden Structure in Liquids, Phys. Rev. A 25, 978 (1982).

[42] F. H. Stillinger and T. A. Weber, Packing structures and transitions in liquids and solids, Science 225, 983 (1984). 
[43] F. H. Stillinger, A topographic view of supercooled liquids and glass formation, Science 267, 1935 (1995).

[44] M. E. Tuckerman, Statistical Mechanics: Theory and Molecular Simulation (Oxford University Press, Oxford, UK, 2010).

[45] B. Bernu and D. M. Ceperley, in Path Integral Monte Carlo, published in Quantum Simulations of Complex Many-Body Systems: From Theory to Algorithms, Lecture Notes, edited by J. Grotendorst, D. Marx, and A. Maramatsu (John von Neumann Institute for Computing, Jülich, 2002), NIC Series, Vol. 10, pp. 51-61.

[46] See Supplemental Material at http://link.aps.org/supplemental/ 10.1103/PhysRevResearch.2.043441 for (i) the definition of the classical FJ pair potential; (ii) criteria for equilibration of the QFJ liquids in the PIMC simulations; (iii) the thermodynamic and PEL properties of the QFJ liquid in the limit $h \rightarrow \infty$; (iv) expressions for the IS energy and pressure for the case of a Gaussian PEL; (v) additional information regarding the structure of the ring-polymers associated with the QFJ liquids; and (vi) the effects of varying $n_{b}$ on our PIMC results.

[47] J. Y. Abraham, S. V. Buldyrev, and N. Giovambattista, Liquid and glass polymorphism in a monatomic system with isotropic, smooth pair interactions, J. Phys. Chem. B 115, 14229 (2011).

[48] S. Reisman and N. Giovambattista, Glass and liquid phase diagram of a polyamorphic monatomic system, J. Chem. Phys. 138, 064509 (2013).

[49] A. Gordon and N. Giovambattista, Glass Transitions in a Monatomic Liquid with Two Glassy States, Phys. Rev. Lett. 112, 145701 (2014).

[50] G. Sun, L. Xu, and N. Giovambattista, Relationship between the potential energy landscape and the dynamic crossover in a water-like monatomic liquid with a liquid-liquid phase transition, J. Chem. Phys. 146, 014503 (2017)

[51] B. Nguyen, G. E. Lopez, and N. Giovambattista, Nuclear quantum effects on the liquid-liquid phase transition of a waterlike monatomic liquid, Phys. Chem. Chem. Phys. 20, 8210 (2018).

[52] Y. Liu, G. Sun, A. Eltareb, G. E. Lopez, N. Giovambattista, and $\mathrm{L}$. Xu, Nuclear quantum effects on the thermodynamic response functions of a polymorphic water-like monatomic liquid, Phys. Rev. Research 2, 013153 (2020).

[53] S. Higuchi, D. Kato, D. Awaji, and K. Kim, Connecting thermodynamic and dynamical anomalies of water-like liquid-liquid phase transition in the Fermi-Jagla model, J. Chem. Phys. 148, 094507 (2018).

[54] P. H. Poole, F. Sciortino, U. Essmann, and H. E. Stanley, Phase behaviour of metastable water, Nature (London) 360, 324 (1992).

[55] O. Mishima and H. E. Stanley, The relationship between liquid, supercooled and glassy water, Nature (London) 396, 329 (1998).

[56] P. G. Debenedetti, Supercooled and glassy water, J. Phys.: Condens. Matter 15, R1669 (2003).

[57] P. Gallo et al., Water: A tale of two liquids, Chem. Rev. 116, 7463 (2016).

[58] P. G. Debenedetti, F. Sciortino, and G. H. Zerze, Second critical point in two realistic models of water, Science 369, 289 (2020).

[59] A. Matsumoto, Parameters of the morse potential from second virial coefficients of gases, Z. Naturforsch. A 42, 447 (1987).

[60] W. H. Press, B. P. Flannery, A. A. Teukolsky et al., Numerical Recipes: The Art of Scientific Computing (Cambridge University Press, Cambridge, UK, 1986).

[61] S. Sastry, P. G. Debenedetti, and F. H. Stillinger, Signatures of distinct dynamical regimes in the energy landscape of a glassforming liquid, Nature (London) 393, 554 (1998).

[62] B. Derrida, Random-energy model: An exactly solvable model of disordered systems, Phys. Rev. B 24, 2613 (1981).

[63] T. Keyes, Entropy, dynamics, and instantaneous normal modes in a random energy model, Phys. Rev. E 62, 7905 (2000).

[64] S. Sastry, The relationship between fragility, configurational entropy and the potential energy landscape of glass-forming liquids, Nature (London) 409, 164 (2001).

[65] A. Heuer and S. Buchner, Why is the density of inherent structures of a lennard-jones-type system gaussian? J. Phys.: Condens. Matter 12, 6535 (2000).

[66] P. H. Handle and F. Sciortino, Potential energy landscape of TIP4P/2005 water, J. Chem. Phys. 148, 134505 (2018). 